acteristic of lymphatic leukemia. It suggests that leukemia, pseudo-leukemia and malignant lymphoma cannot be separated as three distinct entities, and that they do not always differ characteristically in the histological structure of their lymphoid tissues; and that the microscopical sections in these cases will be often wrongly judged in the absence of complete clinical data as regards the distribution of the process and the examination of the blood.

In short, this case emphasizes again our ignorance as to the development of the pathological changes in the diseases of the blood and lymph nodes. It does not warrant any definite conclusions, either specific or general, but is reported simply as a contribution to the literature of these subjects with the hope that it may be of some assistance in the solution of the problems connected with them.

\section{A FURTHER CONTRIBUTION TO THE STUDY OF BACILLURIA IN TYPHOID FEVER AND ITS TREATMENT WITH UROTROPIN.*}

By Chas. D. easton, M.D., Boston.

The literature on the subject of bacilluria in typhoid fever and the use of urotropin in its treatment, up to the year 1899 , may be found in Dr. Mark W. Richardson's paper in the Journal of Experimental Medicine, vol. iv, No. 1, 1899. I have tried to bring the subject up to date. Gwynn ${ }^{1}$ treated successfully 5 cases of cystitis by irrigations with corrosive sublimate 150000 . Two cases were given urotropin. In one the number of bacilli was much diminished but they were still present at the last report. In the second case they finally disappeared after seventeen days. In a later paper ${ }^{2}$ he had 10 cases, in 3 of which the bacilli appeared in the urine before the Widal reaction. He thinks this may be of value in diagnosis. Stress is laid on the importance of urinary disinfection. Urotropin was tried in two of three cases without effect. He prefers mercuric chloride irrigations 1-50000. Further work on the subject ${ }^{3}$ seems to strengthen his former views. He says milk of lime and chlorinated lime are of some value. He also tried formaline, but thinks it is too expensive to come into general use.

Schichold ${ }^{4}$ out of 17 cases found bacilli present in 5 , but thinks that the bacilli are excreted cn'y when the kidney is diseased, beginning immediately as soon as the kidney is affected.

The observations of Walker ${ }^{5}$ and Neufeld ${ }^{6}$ agree with others and both advise the use of urotropin. Walker cites an instance of a well which was infected by the urine of a trooper returning from the army. Twelve persons took the disease.

Horton-Smith ${ }^{7}$ found bacilli in the urine as early as the thirteenth day of the disease, and as late as the fourteenth day of convalescence, and says that they may persist for years.

*Read at the semi-annual meeting of Suffolk District Medical Society, April 29, 1905.
He reports in all 45 cases with 11 of bacilluria. Urotropin was tried in 10 of these and all were cured, but in some cases the bacilli returned and urotropin had to be given again. His idea is that in cystitis the organisms work down into the wall of the bladder.

Lewis ${ }^{8}$ examined 45 cases, but only found bacilli in the urine of 1 case. He speaks well of urotropin and advises its use.

Loida ${ }^{9}$, Schuder ${ }^{10}$, Gehrmann ${ }^{11}$, Klemenko ${ }^{12}$, Vincent ${ }^{13}$ and $\mathrm{Jacobi}^{14}$ agree with other investigators as to the occurrence of bacilli in the urine of their cases. Jacobi reports 643 cases, $27.8 \%$ of which had bacilluria. He did not use urotropin, and considers it of little value because relapses occur and the bacilli return in the urine. Vincent in cystitis uses potassium permanganate irrigations. No other treatment is mentioned in his paper. Neither Klemenko or Schuder used urotropin.

Richardson ${ }^{15}$ reports the finding of the bacilli in the urine as early as the eighth day, while in other cases they were not present until the forty-second day of the disease.

Drake-Brockman ${ }^{18}$ writing on the practical application of urotropin as a urinary antiseptic has seen no untoward results from its administration and thinks it has not received sufficient notice in England.

Ehrmann ${ }^{17}$ used urotropin in a number of affections with success. One case of cystitis was irrigated with silver nitrate and severe hemorrhage followed. Even after bladder irrigations with boracic acid and silver nitrate a cure cannot be expected until urotropin is used in addition. Gordon ${ }^{18}$ reports a case of cerebral syphilis in which urotropin was used and supposed to have caused hematuria. Cammidge ${ }^{10}$ concludes that by the systematic use of urotropin in all cases the very real danger from the urine which is so frequently overlooked may be avoided.

Brown ${ }^{20}$ reports 2 cases of typhoid which he treated with urotropin. One after taking 10 gr., $t . i$. $d$., for two days had painful micturition, and after five days had blood in the urine. The other after $10 \mathrm{gr}$. for seven days had hematuria. Urotropin was omitted and recovery took place in forty-eight hours. Urotropin was given in 13 out of 82 other cases and there was no trouble except in one which was also having turpentine. He believes that the urinary discomfort which precedes hematuria should be considered a danger signal. Millijan ${ }^{21}$ gave 5 gr., $t . i . d$., and in seven days produced pain in the bladder followed by hematuria. Urotropin was stopped and ill effects ceased in two days. Griffith $^{22}$ gave $10 \mathrm{gr}$., $t$. $i$. $d$., and next day there was urinary pain, after ten days backache and swollen eyelids. There was evidently no hematuria in this case. Urotropin was omitted and untoward symptoms ceased after three days. Forbes ${ }^{23}$ also reports a case of pain in bladder and hematuria after $10 \mathrm{gr} ., t . i . d$.

Schumberg ${ }^{24}$ tries to prove that because solutions of urotropin outside the body do not always kill typhoid bacilli that it is of no value 
internally. Clinical experience, however, seems to disprove this theory.

P. Fraenckel, ${ }^{25}$ Orlowski ${ }^{26}$ and Busing ${ }^{27}$ used urotropin with good results. Fraenckel did not make bacteriological examination of the urine of his cases, but gave the drug as a routine measure in the later stages of the disease.

Goetzel and Salus ${ }^{28}$ and Biss ${ }^{20}$ consider urotropin an active antiseptic, especially against typhoid bacilli. Biss has seen 2 cases of hematuria, but thinks it only necessary to be careful in its administration.

Fuchs ${ }^{30}$ found typhoid bacilli in $16 \%$ of cases not treated with urotropin. Of 40 cases in which the drug was given only 1 showed bacilluria. He is of the opinion that even where no bacilli can be found microscopically numerous colonies are obtainable by cultures, and when urotropin is discontinued the bacilli may return in the urine. He thinks the drug must be commenced before the fever subsides, and continued into convalescence to the latest date at which bacilluria is liable to develop.

Knox ${ }^{31}$ advises the treatment with urotropin of all cases of typhoid where the urine is turbid. In no case has he seen any ill effects or discomfort. He considers it of great use and importance in the treatment of typhoid fever in the army.

In an article in the Boston MEDICAL AND Surgical Journal for Feb. 5, 1903, Richardson, without giving the literature, sums up the knowledge on the subject of bacilli in the urine of patients sick with typhoid fever and draws the following conclusions:

" 1 . Typhoid bacilli are present the in urine of about $21 \%$ of individuals affected with typhoid fever.

" 2 . The bacilli when present are usually in pure culture, and their number is frequently enormous: many millions in each cubic centimeter of urine.

" 3 . The invasion of the urine by the bacilli takes place in the later stages of the disease. Unless measures are taken to remove the organisms they persist frequently for weeks, occasionally for months, and rarely for years, and thus constitute $(a)$ a danger to the patient himself, cystitis and possibly orchitis and epididymitis. What is much more important, a grave source of danger to the public health.

" 4 . The necessity for the rigid disinfection and supervision of typhoid urine is apparent.

" 5 . In urotropin we have a drug which will, in the vast majority of cases, remove typhoid organisms from the urine, not only in the cases of simple bacilluria but also in those in which cystitis has resulted. Very rarely an obstinate cystitis may require the use of vesical irrigations. Very infrequently a case will be seen in which the use of urotropin is followed by hematuria. In such cases the drug should be omitted and irrigations of the bladder substituted.

The subject in its relation to the public health is of the utmost importance. In any opinion, it should be a fixed rule, and one rigorously enforced, that no typhoid convalescent be discharged as well until his urine has been proved permanently free from bacilli. In this way only can we prevent a considerable percentage of our typhoid convalescents from becoming unsuspected foci for the further distribution of the disease."

In order to demonstrate the practical value of the above conclusions, during the past year, under Dr. Richardson's direction, and with the consent of the visiting staff, all cases of typhoid fever treated at the Massachusetts General Hospital have been given $5 \mathrm{gr}$. of urotropin three times a day as a routine measure throughout the disease. This was omitted as soon as convalescence was established. In 46 of these cases after the drug had been stopped from six to ten days, and before the patient was discharged, repeated examinations of the urine were made. The specimens were obtained under as nearly as possible aseptic conditions. At first cultures were made on agar plates but it was soon found that the colonies were so few, in many cases none at all, that it was only necessary to use the ayar slant in the culture tubes. In no case of this series was there any growth of typhoid bacilli and all the specimens appeared perfectly clear.

The subsequent history of one of these cases was of particular interest. As soon as the temperature became normal urotropin was omitted. The urine was examined and no typhoid bacilli found. A few days later a relapse began, the urine became slightly cloudy and showed the presence of some cystitis. Large numbers of typhoid bacilli were found in the cultures, urotropin was resumed and he was discharged from the hospital with a clear urine free from bacilli. About one week later patient reported and was found to be suffering from a swollen testicle. There was a slight cystitis and bacilli in the urine. Urotropin was again given, $5 \mathrm{gr}$. three times a day, and five days later the cystitis and bacilluria had disappeared. This case would seem to agree with the theory ${ }^{32}$ that orchitis, epididymitis and prostatis, not uncommon complications of typhoid fever, are ascending infections from the urine containing typhoid bacilli.

In order to determine whether or not the effects of urotropin were permanent, about thirty patients who had been sick with typhoid and treated with urotropin one year previously were sent for. Of this number only ten reported. The examination of the urine of these cases was negative for typhoid organisms.

Coleman ${ }^{33}$ after a careful study and review of the subject concludes that the administration of urotropin may be, but rarely is, attended by toxic effects. I have examined the records of all the cases of typhoid fever treated with urotropin at the Massachusetts General Hospital, comprising 486 cases.

Nearly all received from 8 to 10 grs. three times a day and some 15 gr. three times a day. In some cases the drug was given only two or three days a week, others had it daily. There were 3 cases of painful micturition, and 2 of hematuria. In several cases a few red blood 
corpuscles were found in the microscopical examination of the urine. These all cleared up within a day or two after the withdrawal of the drug. It would thus appear that the danger from toxic action is practically very slight.

It is hardly necessary to-day to call attention to the great need of a simple and effective means of rendering the urine of typhoid patients harmless to themselves and the community. In $1898^{34}$ about one fifth of the soldiers in the national encampments in the United States developed typhoid fever. Among 107,937 officers and men there were 20,738 cases, $19.26 \% \cdot{ }^{34}$ It is believed that in these cases the urine was an important factor in the dissemination of the disease. ${ }^{35}$

The additional conclusions which it would seem justifiable to draw from these investigations are:

1. Although urotropin may, in very rare cases, cause uncomfortable symptoms it does not invalidate the use of the drug.

2. Urotropin is of less value in cases where an active inflammation of the bladder has occurred.

3. But, as far as this series of observations goes, the moderate use of urotropin throughout the disease prevents cystitis.

4. Finally, the routine administration of the drug in all cases of typhoid fever would seem to be strongly indicated, for by such a course of treatment bladder complications are avoided, the urine made inocuous to those brought in contact with the patient, and it is possible to discharge patients who have been sick with typhoid fever in full belief, that, as far as the urine is concerned, they will be harmless to the community.

\section{REFERENCES.}

1 Gwynn: Johns Hop. Hosp. Bull., June, 1899.

2 Gwynn: Phil. Med. Jour., March 3, 1900.

3 Gwynn: Phil. Med. Jour., Jan. 12, 1901.

Schichold: Deutsch. Arch. für Klin. Med., Ixiv, 1899, 505.

S Walker: Brit. Med. Journal, Nov. 24, 1900.

6 Neufeld: Deutsch. Med. Woch., Dec. 20, 1900.

Horton-Smith: Lancet, 1900, 915-1059, Goulstonian Lectures.

8 Lewis: Edin. Med. Jour., Sept., 1901.

Loida: Inaug. Dissert., Konigsberg, 1901.

0 Schuder: Deutsch. Med. Woch., Oct. 31, 1901

1 Gehrmann: Jour. Amer. Asso., Oct. 18, 1902, p. $1,000$.

12 Klemenko: Russ. Archiv. Path., vol. xii, No. 2, Ref. Phil. Med. Jour., 1902 , p. 944.

3 Vincent: Compt. rend. Soc. de Biol., Par., 1903, iv, 365.

$14 \mathrm{Jacobi}$ : Deutsch. Arch. für Klin. Med., 1902, lxxii, 5-6, p. 442.

15 Richardson, Mark: N. Y. State J. M., 1903, iii, 15-33, 21-28.

16 Drake-Brockman: Lond. Lancet, Jan. 30, 1900.

17 Ehrmann: Medical Bulletin, July, 1899.

18 Gordon: Am. Med. Nov. 30, 1901, p. 848

10 Cammidge: Lond. Lancet, 1901, i, 174.

${ }^{20}$ Brown: Brit. Med. Jour., 1901.

21 Millijan: Brit. Med. Jour., June 29, 1901.

22 Griffith: Brit. Med. Jour., June 29, 1901, p. 1617

23 Forbes: Brit. Med. Jour., June 29, 1901.

Schumburg: Deutsch. Med. Woch., Nov. 9, 1901

25 P. Fraenckel: Deutsch. med. Woch., March 28, 1901

26 Orlowski: (Russian) Ref. Cent. für Bakt., xxix, 227.

2 Büsing: Deutsch. Med. Woch. June 19, 1902.

${ }^{28}$ Goetzel \& Salus: Zeit. für K1. Med., xlv, Hft., 516

Biss: Edin. Med. Jour., October, 1902.

1903.

${ }_{31}$ Knox: J. Ry. Army M. Corp., Lond., 1904, iii, 1-16.

32 Richardson, Mark: Address rend. at Annual Meeting, Mass

Med. Soc., June, 1898 .

${ }_{33}$ Coleman: N. Y. Med. News, 1903, 83-393-399.

34 Reed, Vaughan \& Shakespeare: Report on Typhoid Fever

During Spanish War, 1904 , vol. i, p. 647.

The first International Congress of Radiology and Ionization will take place at Liège, Belgium, from September 12 to 14 .

\section{apedital erogress.}

REPORT ON OBSTETRICS.
BX FRANK A. HIGGINs, M.D., BOsTON.

VAGINAL CESAREAN SECTION IN ACCOUCHEMENT FORCE.

ZiNKE, ${ }^{1}$ in a paper on the " Relative Value of the Means and Methods Employed in Accouchement Forcé," recommends very highly the method of deep cervical incisions, or the so-called vaginal Cesarean section, and he believes they are destined to play a permanent and important rôle in the management of forced labors in the future. Many of the cases now subjected to manual or balloon dilatation will be treated by cervical incisions. He says it is the method in the presence of sepsis of the vagina, because the operation is short in duration and can be performed under a continuous flow of an antiseptic solution. An intact cervix, whether hard or elongated or not, is always an indication for cervical incision; also when the cervix is the site of malignant disease or extensive cicatrization. Cervical incisions are also recommended in premature rupture of the membranes, generally contracted pelves, elcampsia, heart disease, articulo mortis, threatened rupture of the uterus, placenta previa, albatio placentæ, and other conditions demanding rapid delivery.

Carstens ${ }^{1}$ also recommends the vaginal Cesarean operation for delivery in grave cases of eclampsia. as he believes it enables the patient to be delivered quickly and safely. Deep lateral incisions he regards as dangerous, as in one of his cases in which this was done the wounds were extended by tearing into the broad ligament, in the delivery of the fetus, so that much hemorrhage is likely to occur. He reports two cases following the technic of Dührssen, making one median anterior incision through the cervix and as far as is necessary in the uterus, first separating the bladder up as far as the peritoneum by a transverse incision at the junction of the anterior vaginal wall with the cervix, - the bladder is then easily pushed up out of the way. There is very little hemorrhage and the wound is easily sutured. The patient is delivered by forceps after the incision is made.

He says the operation is not difficult and any surgeon having had some experience in vaginal work can have no trouble with a prompt and sure delivery and in saving the patient.

\section{NON-OPERATIVE TREATMENT OF ECLAMPSIA.}

Bacon ${ }^{2}$ emphasizes the importance of rest in bed and a milk diet in the medical treatment of eclampsia. In other respects he differs from what has been generally accepted as good treatment. He says the recent theories concerning the action of sodium chloride and other similar salts in cases of nephritis and edema, which are causing a considerable change in the method of treating these diseased conditions, are of much interest to us because of their bearing on the 1 Am. Jour. Obstetrics, November, 1904.

2 Am. Jour. Obstetrics, November, 